This item was submitted to Loughborough's Research Repository by the author.

Items in Figshare are protected by copyright, with all rights reserved, unless otherwise indicated.

\title{
Adaptive fuzzy tracking control for a class of uncertain MIMO nonlinear systems using disturbance observer
}

PLEASE CITE THE PUBLISHED VERSION

http://dx.doi.org/10.1007/s11432-012-4695-3

PUBLISHER

(C) Science China Press and Springer-Verlag

VERSION

AM (Accepted Manuscript)

LICENCE

CC BY-NC-ND 4.0

REPOSITORY RECORD

Chen, Mou, Wen-Hua Chen, and Qing-Xian Wu. 2012. "Adaptive Fuzzy Tracking Control for a Class of Uncertain MIMO Nonlinear Systems Using Disturbance Observer”. figshare. https://hdl.handle.net/2134/11169. 
This item was submitted to Loughborough's Institutional Repository (https://dspace.lboro.ac.uk/) by the author and is made available under the following Creative Commons Licence conditions.

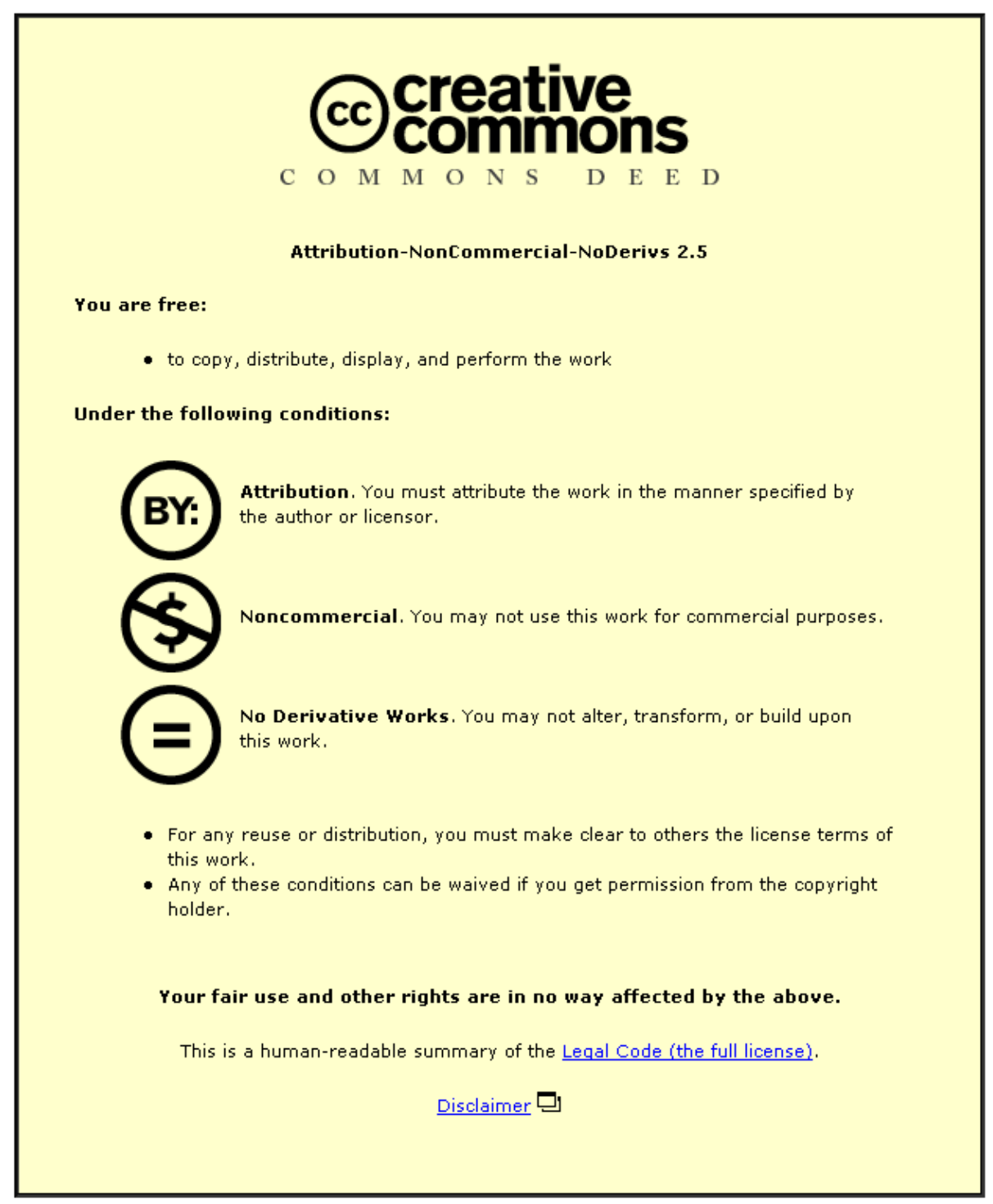

For the full text of this licence, please go to: http://creativecommons.org/licenses/by-nc-nd/2.5/ 


\title{
Adaptive Fuzzy Tracking Control for a Class of Uncertain MIMO Nonlinear Systems Using Disturbance Observer
}

\author{
Mou Chen ${ }^{1 *}$ and Wen-hua Chen ${ }^{2}$ \\ College of Automation Engineering \\ Nanjing University of Aeronautics and Astronautics \\ Nanjing, China 210016 \\ ${ }^{2}$ Department of Aeronautical and Automotive Engineering \\ Loughborough University \\ Loughborough, Leicestershire LE11 3TU, UK
}

\begin{abstract}
In this paper, adaptive fuzzy tracking control is proposed for a class of multi-input and multi-output (MIMO) nonlinear systems in the presence of system uncertainties, unknown nonsymmetric input saturation and unknown external disturbances. For the MIMO nonlinear system, the time-varying external disturbance cannot be directly measured which is estimated via disturbance observer. By choosing the appropriate gain matrix, disturbance observer can approximate the unknown disturbance well and the estimate error converges to a compact set. And then, fuzzy logic systems (FLS) are used to approximate the system uncertainty of MIMO nonlinear systems. Based on outputs of disturbance observer and FLS, adaptive tracking control is presented for the uncertain MIMO nonlinear system. Considering the unknown non-symmetric input saturation and control singularity case, adaptive fuzzy tracking control is developed via disturbance observer and FLS approximation technique. The effects of unknown input saturation and control singularity are treated as a part of system compound disturbance which is estimated using disturbance observer. Under the proposed tracking control techniques, semi-global uniform boundedness of the closed-loop signals is guaranteed via Lyapunov analysis. Numerical simulation results are presented to illustrate the effectiveness of the proposed tracking control schemes.
\end{abstract}

Keywords: MIMO nonlinear system, Input saturation, Disturbance observer, Fuzzy logic systems, Tracking control

\section{Introduction}

In the past years, robust adaptive control of uncertain nonlinear systems has received much attention to enhance performance robustness of the closed-loop control system. In practice, most control plants

${ }^{*}$ Corresponding author. Tel: $(+86)+25+84893084$, Fax: $(+86)+25+84892300$, E-mail: chenmou@nuaa.edu.cn. 
are multivariable and are characterized by widely changing environmental disturbances and various work conditions. Thus, it is important to investigate effective robust adaptive control techniques for uncertain MIMO nonlinear systems [1-7]. Robust adaptive control based on universal function approximators (such as fuzzy systems and neural networks) has been extensively studied [8-12]. In particular, adaptive fuzzy control has been an active impact in the control community because adaptive fuzzy control provides a systematic and efficient framework to incorporate linguistic fuzzy information from human experts [13-16]. In [17], adaptive fuzzy robust tracking control and and its application were studied via small gain approach. Direct adaptive output-feedback fuzzy control was designed for the nonaffine nonlinear system [18]. In [19], fuzzy control design was given for the trajectory tracking on uncertain nonlinear systems. Adaptive fuzzy-based tracking control was proposed for nonlinear systems via VSS and $H_{\infty}$ approaches in [20]. In [21], robust adaptive tracking control was studied for nonlinear systems based on bounds of fuzzy approximation parameters. Adaptive fuzzy output tracking control was proposed for a class of uncertain nonlinear systems in [22]. However, the time-varying external disturbance of the nonlinear system cannot be efficiently tackled via fuzzy logic systems. To improve the anti-disturbance ability of the closed-loop control system, the disturbance-observer-based control can be developed in which disturbance observer is adopted to estimate the unknown external disturbance.

In the recent decade, various design techniques have been intensively developed for disturbance observer to fully use the information of external disturbances [23-27]. In literatures, disturbance observers were used to approximate external disturbance, and robust control were developed based on the output of disturbance observers. In $[24,25]$, fuzzy disturbance observer and its application were studied for discrete-time and continuous-time systems. The general framework was given for nonlinear systems subject to disturbances using disturbance observer based control (DOBC) technique [26]. The nonlinear predictive control was proposed using the disturbance observer in [27]. In [28], nonlinear-disturbance observer was proposed for multivariable minimum-phase systems with arbitrary relative degrees. The nonlinear disturbance observer for robotic manipulators was developed in [29]. In [30], composite disturbance-observer-based control and terminal sliding mode control (TSMC) were investigated for uncertain structural systems. In [31], nonlinear disturbance observerbased approach was proposed for longitudinal dynamics of a missile. The disturbance attenuation and rejection problem was investigated for a class of MIMO nonlinear systems using DOBC framework in [32]. In [33], composite DOBC and $H_{\infty}$ control were proposed for complex continuous models. Composite DOBC and TSMC were developed for nonlinear systems with disturbances in [34]. In [35], sliding mode synchronization control was proposed for uncertain chaotic systems based on distur- 
bance observer. Sliding mode control was developed for a class of uncertain nonlinear system using disturbance observer in [36]. In [37], robust DOBC was presented for time delay uncertain systems. However, the disturbance observer should be further developed for the uncertain MIMO system with input saturation.

Input saturation is the most important non-smooth nonlinearity which should be explicitly considered in the control design. The analysis and design of control systems with input saturation constraints have been widely studied [38-42]. In [39], tracking control was developed for ocean surface vessels with input saturation using the neural network. Robust adaptive neural network control was proposed for a class of uncertain MIMO nonlinear systems with input nonlinearities [38]. Hover control was studied for an unmanned aerial vehicle (UAV) with input saturations in [40]. In [41], robust stability analysis and fuzzy-scheduling control were developed for nonlinear systems with input saturation. Globally stable adaptive control was presented for minimum phase single-input and single-output (SISO) plants with input saturation [42]. Nonlinear control was proposed to obtain the attitude maneuver of a three-axis stabilized flexible spacecraft considering input nonlinearity [43]. It is apparent that the input saturation constraint will enhance the complexity of control system design for the uncertain MIMO nonlinear system. Furthermore, robust control for uncertain MIMO nonlinear systems with control singularity case is a promising research topic. Thus, adaptive fuzzy tracking control need to be further developed for the uncertain MIMO nonlinear system with control singulary case and unknown non-symmetric input saturation.

This work is motivated by adaptive fuzzy tracking control of uncertain MIMO systems with system uncertainties, unknown external disturbance and unknown non-symmetric input saturation. The control objective is to track a desired trajectory in the presence of systems uncertainties, the time varying disturbances and input saturation. The main contributions of this paper are as follows:

(i) nonlinear disturbance observer is developed to approximate the unknown external disturbance for the uncertain MIMO nonlinear system and the bounded approximation error is guaranteed.

(ii) to the best of my knowledge, it is the first time in the literature that the disturbance-observerbased tracking control is developed for the uncertain MIMO nonlinear system with external disturbance, control singularity and unknown non-symmetric input saturation.

(iii) rigorous stability of the disturbance-observer-based tracking control is guaranteed using Lyapunov analysis which shows that the semiglobal uniform boundedness of all closed-loop signals.

The organization of the paper is as follows. The system statement and preliminaries are given 
in Section 2. Section 3 describes the design of nonlinear disturbance observer. Adaptive fuzzy tracking control is proposed based on disturbance observer in Section 4. In Section 5, adaptive fuzzy tracking control is developed for MIMO nonlinear systems with unknown input saturation and control singularity. Simulation studies are shown in Section 6 to demonstrate the effectiveness of our proposed control approaches, followed by some concluding remarks in Section 7.

\section{Problem Formulation and Preliminary}

\subsection{Problem Formulation}

Consider the uncertain MIMO nonlinear system described in the form of

$$
y^{(n)}=F\left(y^{(n-1)}, \ldots, \dot{y}, y\right)+\Delta F\left(y^{(n-1)}, \ldots, \dot{y}, y\right)+G\left(y^{(n-1)}, \ldots, \dot{y}, y\right) u+d\left(y^{(n-1)}, \ldots, \dot{y}, y, t\right)
$$

where $y \in R^{n}$ is the system output vector; $F(.) \in R^{n}$ is a known nonlinear function vector; $G(.) \in$ $R^{n \times m}$ is a known nonlinear function matrix; $\Delta F\left(y^{(n-1)}, \ldots, \dot{y}, y\right) \in R^{n}$ is a unknown nonlinear function vector which denotes the system uncertainty; $d\left(y^{(n-1)}, \ldots, \dot{y}, y\right) \in R^{n}$ is the time-varying external disturbance of the uncertain MIMO nonlinear system (1).

For further facilitate proceed the adaptive fuzzy control design, we define

$$
x_{1}=y, x_{2}=\dot{y}, \ldots, x_{n}=y^{(n-1)}
$$

According to (2), the uncertain nonlinear system (1) can be transformed into the following system:

$$
\begin{aligned}
\dot{x}_{i} & =x_{i+1}, \quad i=1,2, \ldots, n-1 \\
\dot{x}_{n} & =F(x)+G(x) u+\Delta F(x)+d(x, t) \\
y & =x_{1}
\end{aligned}
$$

where $x=\left[x_{1}, x_{2}, \ldots, x_{n}\right]^{T}$ is measurable.

To develop the disturbance-observer-based adaptive fuzzy tracking control for the uncertain MIMO nonlinear system (1), the following assumption and lemma are required:

Lemma 1 [44] For bounded initial conditions, if there exists a $C^{1}$ continuous and positive definite Lyapunov function $V(x)$ satisfying $\pi_{1}(\|x\|) \leq V(x) \leq \pi_{2}(\|x\|)$, such that $\dot{V}(x) \leq-\kappa V(x)+c$, where $\pi_{1}, \pi_{2}: R^{n} \rightarrow R$ are class $K$ functions and $c$ is a positive constant, then the solution $x(t)$ is uniformly bounded. 
In this paper, the control objective is to develop the disturbance-observed-based adaptive fuzzy tracking control to such that all closed-loop system signals are asymptotically convergent in the presence of unknown input saturation and the time-varying external disturbance and ensure that the system output track the desired signal. The desired tracking signal $y_{d}$ satisfies the following assumption:

Assumption 1 For all $t>0$, there exist $\Delta>0$ such that $\left\|y_{d}^{(n)}(t)\right\| \leq \Delta$.

\subsection{Fuzzy Logical Systems}

In this paper, fuzzy logic systems are chosen to approximate unknown function term $\Delta F(x)$ of the uncertain MIMO nonlinear system (3). The fuzzy inference engine uses fuzzy IF-THEN rules to perform a mapping from an input linguistic vector $Z=\left[z_{1}, z_{2}, \ldots, z_{l}\right]^{T} \in R^{l}$ to an output variable $Y \in R$. Then, the $i t h$ fuzzy rule can be represented as [5]:

$$
R^{i} \text { : If } z_{1} \text { is } A_{1}^{i} \text { and } \ldots \text { and } z_{l} \text { is } A_{l}^{i} \text {, then } Y \text { is } Y^{i}
$$

where $A_{1}^{i}, A_{2}^{i}, \ldots, A_{l}^{i}$ and $Y^{i}$ are fuzzy sets characterized using fuzzy membership functions.

The output of the fuzzy system with the singleton fuzzifier, product inference engine and centeraverage defuzzifier can be expressed as

$$
Y(Z)=\frac{\sum_{i=1}^{r} Y^{i}\left(\prod_{j=1}^{l} \mu_{A_{j}^{i}}\left(z_{j}\right)\right.}{\prod_{j=1}^{l} \mu_{A_{j}^{i}}\left(z_{j}\right)}=\hat{W}^{T} \varphi(Z)
$$

where $\mu_{A_{j}^{i}}\left(z_{j}\right)$ is the membership function value of fuzzy variables $z_{j}$, and $r$ is the number of fuzzy rules. $\hat{W}^{T}=\left(Y^{1}, Y^{2}, \ldots, Y^{r}\right)$ is an adjustable parameter vector. $\varphi=\left(\varphi_{1}, \varphi_{2}, \ldots, \varphi_{r}\right)$, and $\varphi_{i}=$ $\frac{\prod_{j=1}^{l} \mu_{A_{j}^{i}}\left(z_{j}\right)}{\sum_{i=1}^{r}\left(\prod_{j=1}^{n} \mu_{A_{j}^{i}}\left(z_{j}\right)\right)}$ are fuzzy basis functions. Hence, the approximation of uncertain function term $f(Z)$ can be expressed as

$$
\hat{f}(Z)=\hat{W}^{T} \varphi(Z)
$$

where $Z \in \Omega_{Z} \subset R^{q}, \hat{W}=\left[\hat{W}_{1}, \hat{W}_{2}, \ldots, \hat{W}_{l}\right] \in R^{l \times l}, \varphi(Z)=\left[\varphi_{1}, \varphi_{2}, \ldots, \varphi_{l}\right]^{T} \in R^{l \times 1}$.

If $Z$ belong to a compact set $M_{Z}$, the optimal parameter vector $W^{*}$ is defined as

$$
W^{*}=\arg \min _{\hat{W} \in M_{W}}\left[\sup _{Z \in M_{Z}}|f(Z)-\hat{f}(Z \mid \hat{W})|\right]
$$

where the parameter vector $\hat{W}$ lies in a convex region given by

$$
M_{W}=\left\{\hat{W} \mid\|\hat{W}\| \leq m_{W}\right\}
$$


where $m_{W}$ is a design parameter.

Under the optimization parameter vector, the unknown uncertain term $f(Z)$ can be written as

$$
f(Z)=W^{* T} \varphi(Z)+\varepsilon
$$

where $\varepsilon=\left[\varepsilon_{1}, \varepsilon_{2}, \ldots, \varepsilon_{l}\right]^{T}$ is the smallest approximation error of FLS. It is apparent that we have

$$
\|\varepsilon\| \leq \varepsilon^{*}
$$

where $\varepsilon^{*}>0$ is the upper bound of approximation error.

\section{Design of Nonlinear Disturbance Observer}

In the MIMO nonlinear system (3), the unknown nonlinear term $\Delta F(x)$ is approximated via fuzzy logic systems. Considering (3) and (9), we have

$$
\dot{x}_{n}=F(x)+G(x) u+W^{* T} \varphi(Z)+\varepsilon+d(x, t)
$$

where $\varepsilon$ is the approximation error of fuzzy logic system which is unknown and $Z=\left[x_{1}, x_{2}, \ldots, x_{n}\right]^{T}$. To efficiently tackle the unknown approximation error $\varepsilon$, it can be treated as a part of the system external disturbance. Define $D=\varepsilon+d$. Then, equation (11) can be expressed as

$$
\dot{x}_{n}=F(x)+G(x) u+W^{* T} \varphi(Z)+D
$$

Since $D$ is unknown in (12), it cannot be directly used to develop the adaptive fuzzy tracking control for the uncertain MIMO nonlinear system (3). Thus, we design the disturbance observer to estimate it. To proceed the design of disturbance observer, we assume that the disturbance $D$ is time-varying but with bounded variation. Thus, we have

$$
\|\dot{D}\| \leq \rho
$$

where $\rho$ is an unknown positive constant.

To design a nonlinear disturbance observer, an auxiliary variable is introduced as follows.

$$
z=D-K x_{n}
$$

where $K=K^{T}>0$ is a design matrix. 
Considering (12), the time derivative of $z$ can be written as

$$
\begin{aligned}
\dot{z} & =\dot{D}-K \dot{x}_{n} \\
& =\dot{D}-K\left(F(x)+G(x) u+W^{* T} \varphi(Z)+D\right) \\
& =\dot{D}-K\left(F(x)+G(x) u+W^{* T} \varphi(Z)+z+K x_{n}\right)
\end{aligned}
$$

To obtain the disturbance estimate, the estimate of intermediate variable $z$ is presented as

$$
\dot{\hat{z}}=-K\left(F(x)+G(x) u+\hat{z}+K x_{n}\right)
$$

where $\hat{z}$ is the estimate of $z$.

Invoking (12), the estimate of disturbance $D$ can be written as

$$
\hat{D}=\hat{z}+K x_{n}
$$

Define

$$
\tilde{D}=D-\hat{D}=z-\hat{z}=\tilde{z}
$$

Differentiating (18), and considering (15) and (16) yields

$$
\dot{\tilde{z}}=\dot{z}-\dot{\hat{z}}=\dot{D}-K \tilde{z}-K W^{* T} \varphi(Z)
$$

The design of nonlinear disturbance observer can be summarized in the following theorem which contains the detailed design process of disturbance observer.

Theorem 1 Considering the uncertain MIMO nonlinear system (3), the nonlinear disturbance observer is designed as (16) and (17). Then, the approximation error of the proposed nonlinear disturbance observer is bounded. Namely, the approximation error $\tilde{D}$ remains within the compact sets $\Omega_{\tilde{D}}$ defined by

$$
\Omega_{\tilde{D}}:=\left\{\tilde{D} \in R^{m} \mid\|\tilde{D}\| \leq \sqrt{\Omega_{0}}\right\}
$$

where $\Omega_{0}=2\left(V_{o}(0)+\frac{C_{0}}{\kappa_{0}}\right)$ with $C_{0}$ and $\kappa_{0}$ as defined in (24).

Proof: To analyze the convergent ability of disturbance estimate error $\tilde{D}$, the Lyapunov function candidate can be written as

$$
V_{o}=\frac{1}{2} \tilde{D}^{T} \tilde{D}=\frac{1}{2} \tilde{z}^{T} \tilde{z}
$$


Considering (19), the derivative of $V_{o}$ is

$$
\dot{V}_{o}=\tilde{z}^{T} \dot{D}-\tilde{z}^{T} K \tilde{z}-\tilde{z}^{T} K W^{* T} \varphi(Z)
$$

Considering the expression of the fuzzy basis function, we have

$$
\|\varphi(Z)\| \leq \xi
$$

where $\xi>0$

Invoking (21), we obtain

$$
\begin{aligned}
\dot{V}_{o} & \leq-\tilde{z}^{T}\left(K-I_{m \times m}\right) \tilde{z}+\frac{1}{2} \xi\|K\|\left\|W^{*}\right\|+\frac{1}{2}\|\dot{D}\| \\
& \leq-\tilde{z}^{T}\left(K-I_{m \times m}\right) \tilde{z}+\frac{1}{2} \xi\|K\|\left\|W^{*}\right\|+\frac{1}{2} \rho^{2} \\
& \leq-\kappa_{0} V_{o}+C_{0}
\end{aligned}
$$

where

$$
\begin{aligned}
\kappa_{0}: & =\lambda_{\min }\left(K-I_{m \times m}\right) \\
C_{0}: & =\frac{1}{2} \xi\|K\|\left\|W^{*}\right\|+\frac{1}{2} \rho^{2}
\end{aligned}
$$

To ensure the closed-loop system stability, the disturbance observer gain matrix $K$ should be chosen to make $K-I_{m \times m}>0$. According to (23) and Lemma 1, it can directly show that the signals $\tilde{D}$ and $\tilde{z}$ are semiglobally uniformly bounded. For completeness, the detailed proof are presented here. Multiplying (23) by an exponent function $\exp \left(\kappa_{0} t\right)$, there yields $\frac{d}{d t}\left(V_{o}\right) \exp \left(\kappa_{0} t\right)$. Integration of this equation yields

$$
V_{o} \leq\left(\left.V_{o}\right|_{t=0}-\frac{C_{0}}{\kappa_{0}}\right) \exp \left(-\kappa_{0} t\right)+\frac{C_{0}}{\kappa_{0}} \leq\left. V_{o}\right|_{t=0}+\frac{C_{0}}{\kappa_{0}}
$$

Considering (20) and (25), we have

$$
\frac{1}{2}\|\tilde{D}\|^{2} \leq\left. V_{o}\right|_{t=0}+\frac{C_{0}}{\kappa_{0}} \Rightarrow\|\tilde{D}\| \leq \sqrt{2\left(\left.V_{o}\right|_{t=0}+\frac{C_{0}}{\kappa_{0}}\right)}
$$

Thus, the approximation error $\tilde{D}$ of the developed disturbance observer is bounded. This concludes the proof. $\diamond$

Remark 1 Since many practical nonlinear systems possesses the unknown time-varying external disturbance, the nonlinear disturbance observer is developed to monitor external disturbance in this paper. To the proposed disturbance observer, we can see that the approximation error with suitable transient performance can be obtained by adjusting the gain matrix $K$ of disturbance observer. However, caution must be exercised in the choice of gain matrix of disturbance observer, due to the fact that there are some trade-off between the disturbance approximation performance and other issues. 


\section{Adaptive Fuzzy Control Design Based on Disturbance Observer}

In this section, we develop adaptive fuzzy tracking control scheme for the uncertain MIMO nonlinear system (3) using the developed disturbance observer and fuzzy logic systems. To propose the disturbance-observer-based adaptive fuzzy tracking control, the tracking error is defined as

$$
e=y_{d}-y
$$

Based on the tracking error $e$, we define

$$
e_{s}=e^{(n-1)}(t)+k_{n-2} e^{(n-2)}(t)+\ldots+k_{1} \dot{e}(t)+k_{0} e(t)
$$

where design parameters $k_{0}, k_{1}, \ldots, k_{n-2}$ are chosen as positive constants such that the polynomial $s^{n-1}+k_{n-2} s^{n-2}+\ldots+k_{0}$ is Hurwitz.

Considering (27) and (28), $\bar{e}_{s}$ is given by

$$
\bar{e}_{s}=\dot{e}_{s}-e^{(n)}
$$

To develop adaptive fuzzy tracking control for the uncertain MIMO nonlinear system (3), we assume that $G(x)$ is nonsingular [45]. Under this assumption, the disturbance-observed-based adaptive fuzzy tracking control is proposed as

$$
u=G^{-1}(x)\left(-F(x)-\hat{W}^{T} \varphi(Z)+\tau-\hat{D}\right)
$$

where $\tau$ is given by

$$
\tau=y_{d}^{(n)}+\gamma e_{s}+\bar{e}_{s}
$$

where $\gamma>0$.

Substituting (30) into (12), we obtain

$$
\begin{aligned}
y^{(n)} & =F(x)+G(x) u+\Delta F(x)+d \\
& =W^{* T} \varphi(Z)-\hat{W}^{T} \varphi(Z)+\tau+D-\hat{D} \\
& =-\tilde{W}^{T} \varphi(Z)+\tau+\tilde{D}
\end{aligned}
$$

where $\tilde{W}=\hat{W}-W^{*}$.

Considering (31), equation (32) can be rewritten as

$$
\dot{e}_{s}=-\gamma e_{s}+\tilde{W}^{T} \varphi(Z)-\tilde{D}
$$


Consider the adaptive law for $\hat{W}$ as

$$
\dot{\hat{W}}=-\Lambda\left(\varphi(Z) e_{s}^{T}+\beta \hat{W}\right)
$$

where $\Lambda=\Lambda^{T}>0$ and $\beta>0$.

The above design procedure can be summarized in the following theorem, which contains the results of disturbance-observer-based adaptive fuzzy tracking control for the system dynamics (3) with the unknown time-varying external disturbance.

Theorem 2 Consider the uncertain MIMO nonlinear system (3) with the unknown time-varying external disturbance $D$ which is estimated via disturbance observer (16) and (17). Then, all closedloop system signals are semi-globally uniformly stable under the proposed disturbance-observer-based adaptive fuzzy tracking control (30). Furthermore, the tracking error e, the disturbance approximation error $\tilde{D}$ and the parameter estimate error $\tilde{W}$ of fuzzy system remain within the compact sets $\Omega_{e}, \Omega_{\tilde{D}}$ and $\Omega_{\tilde{W}}$ respectively, defined by

$$
\begin{aligned}
\Omega_{e} & :=\left\{e \in R^{n} \mid\|e\| \leq \sqrt{\Omega_{1}}\right\} \\
\Omega_{\tilde{D}} & :=\left\{\tilde{D} \in R^{n} \mid\|\tilde{D}\| \leq \sqrt{\Omega_{1}}\right\} \\
\Omega_{\tilde{W}} & :=\left\{\tilde{W} \in R^{l} \mid\|\tilde{W}\| \leq \sqrt{\frac{\Omega_{1}}{\lambda_{\max }\left(\Lambda^{-1}\right)}}\right\}
\end{aligned}
$$

where $\Omega_{1}=2\left(V(0)+\frac{C}{\kappa}\right)$ with $C$ and $\kappa$ as defined in (39).

Proof: Let the Lyapunov function candidate be given by

$$
V=\frac{1}{2} e_{s}^{T} e_{s}+\frac{1}{2} \tilde{D}^{T} \tilde{D}+\frac{1}{2} \operatorname{tr}\left(\tilde{W}^{T} \Lambda^{-1} \tilde{W}\right)
$$

The time derivative of $V$ along the state trajectory is

$$
\begin{aligned}
\dot{V} & =e_{s}^{T} \dot{e}_{s}+\tilde{D}^{T} \dot{\tilde{D}}+\operatorname{tr}\left(\tilde{W}^{T} \Lambda^{-1} \dot{\tilde{W}}\right)=e_{s}^{T} \dot{e}_{s}+\tilde{z}^{T} \dot{\tilde{z}}+\operatorname{tr}\left(\tilde{W}^{T} \Lambda^{-1} \dot{\tilde{W}}\right) \\
& \leq-\gamma e_{s}^{T} e_{s}+e_{s}^{T} \tilde{W}^{T} \varphi(Z)-e_{s}^{T} \tilde{D}+\tilde{z}^{T} \dot{D}-\tilde{z}^{T} K \tilde{z}-\tilde{z}^{T} K W^{* T} \varphi(Z)+\operatorname{tr}\left(\tilde{W}^{T} \Lambda^{-1} \dot{\tilde{W}}\right)
\end{aligned}
$$

Considering the updated law (34) of the fuzzy system parameter and the following fact

$$
2 \operatorname{tr}\left(\tilde{W}^{T} \hat{W}\right)=\|\tilde{W}\|^{2}+\|\hat{W}\|^{2}-\left\|W^{*}\right\|^{2} \geq\|\tilde{W}\|^{2}-\left\|W^{*}\right\|^{2}
$$

we obtain

$$
\begin{aligned}
\dot{V} & \leq-\gamma e_{s}^{T} e_{s}-e_{s}^{T} \tilde{D}+\tilde{z}^{T} \dot{D}-\tilde{z}^{T} K \tilde{z}-\tilde{z}^{T} K W^{* T} \varphi(Z)-\frac{\beta}{2}\|\tilde{W}\|^{2}+\frac{\beta}{2}\left\|W^{*}\right\|^{2} \\
& =-\gamma e_{s}^{T} e_{s}-e_{s}^{T} \tilde{z}+\tilde{z}^{T} \dot{D}-\tilde{z}^{T} K \tilde{z}-\tilde{z}^{T} K W^{* T} \varphi(Z)-\frac{\beta}{2}\|\tilde{W}\|^{2}+\frac{\beta}{2}\left\|W^{*}\right\|^{2} \\
& \leq-(\gamma-0.5)\left\|e_{s}\right\|^{2}-\tilde{z}^{T}\left(K-1.5 I_{m \times m}\right) \tilde{z}-\frac{\beta}{2}\|\tilde{W}\|^{2}+\frac{1}{2} \xi\|K\|\left\|W^{*}\right\|+\frac{\beta}{2}\left\|W^{*}\right\|^{2}+\frac{1}{2} \rho^{2} \\
& \leq-\kappa V+C
\end{aligned}
$$


where

$$
\begin{aligned}
\kappa: & =\min \left((\gamma-0.5), \lambda_{\min }\left(K-1.5 I_{m \times m}\right), \frac{2 \beta}{\lambda_{\max }\left(\Lambda^{-1}\right)}\right) \\
C: & =\frac{1}{2} \xi\|K\|\left\|W^{*}\right\|+\frac{1}{2} \rho^{2}
\end{aligned}
$$

To ensure the closed-loop system stability, the corresponding design parameters $\gamma, K$ and $\beta$ should be chosen to make $\gamma-0.5>0$ and $K-I_{m \times m}>0$. According to (39) and Lemma 1, it can directly show that the signals $\tilde{D}, e$ and $\tilde{W}$ are semiglobally uniformly bounded. For completeness, the details of the proof are omitted here. This concludes the proof. $\diamond$

Remark 2 In the developed adaptive fuzzy tracking control, the output of nonlinear disturbance observer is adopted. Thus, the upper boundary of the unknown external disturbance is not required in the design of adaptive fuzzy tracking control (30). Furthermore, the proposed disturbance-observerbased adaptive fuzzy tracking control can fully explore the dynamic characteristic of the time-varying disturbances. It is worth to point out that the convergence performance of the tracking error e, the disturbance approximation error $\tilde{D}$ and the parameter estimate error $\tilde{W}$ depends on the choice of design parameters $\gamma, K$ and $\beta$.

\section{Adaptive Fuzzy Control Design Considering Unknown Input Sat- uration and Control Singularity}

In Section 4, adaptive fuzzy tracking control has been developed for the uncertain MIMO nonlinear system based on disturbance observer. However, control gain matrix $G(x)$ has been required nonsingular. Since $G(x)$ depends on the system state $x$, there exists the singular feasibility at a special moment in the practical system, i.e, $|G(x)|=0$. On the other hand, input saturation has not been considered. In fact, input saturation always exists due to actuator output constraint. If input saturation is ignored in the control design, the closed-loop control system performance may be degraded. Therefore, adaptive fuzzy tracking control design will be presented for the uncertain MIMO nonlinear systems with control singularity and unknown input saturation in this section.

Considering the uncertain MIMO nonlinear system (3) with unknown non-symmetric input saturation, the control input $u=\left[u_{1}, u_{2}, \ldots, u_{m}\right]^{T}$ is given by

$$
u_{i}= \begin{cases}u_{i \max }, & \text { if } v_{i}>u_{i \max } \\ v_{i}, & \text { if }-u_{i \min } \leq v_{i} \leq u_{i \max } \\ -u_{i \min }, & \text { if } v_{i}<-u_{i \min }\end{cases}
$$


where $v=\left[v_{1}, v_{2}, \ldots, v_{m}\right]^{T}$ is the designed control input demand. $u_{i \min }$ and $u_{i \max }$ are the unknown parameters of non-symmetric input saturation.

To efficiently tackle and analyze the control singularity and unknown input saturation, the adaptive fuzzy tracking control is proposed as

$$
v=G^{T}(x)\left(G(x) G^{T}(x)+\delta I\right)^{-1} u_{r}
$$

where $\delta$ is a positive design parameter.

It is apparent that we have

$$
G(x) G^{T}(x)\left(G(x) G^{T}(x)+\delta I\right)^{-1}=I-\delta\left(G(x) G^{T}(x)+\delta I\right)^{-1}
$$

Considering (42), and substituting (41) into (12), we obtain

$$
\begin{aligned}
\dot{x}_{n} & =F(x)+G(x) u+\Delta F(x)+d(x, t) \\
& =F(x)+G(x)(v+\Delta u)+W^{* T} \varphi(Z)+D \\
& =F(x)+u_{r}+W^{* T} \varphi(Z)+D+G(x) \Delta u-\delta\left(G(x) G^{T}(x)+\delta I\right)^{-1} v
\end{aligned}
$$

where $\Delta u=u-v$.

Since $\Delta u$ is unknown, it can be treated as a part of the disturbance. Define the compound disturbance as follows:

$$
\bar{D}=D+G(x) \Delta u-\delta\left(G(x) G^{T}(x)+\delta I\right)^{-1} v
$$

Considering (44), we have

$$
\dot{x}_{n}=F(x)+u_{r}+W^{* T} \varphi(Z)+\bar{D}
$$

Due to the unknown $\bar{D}$, the disturbance observer is used to approximated it. Similar with (16), the observer of the intermediate variable $z$ can be designed as

$$
\dot{\hat{z}}=-K\left(F(x)+u_{r}+\hat{z}+K x_{n}\right)
$$

where $K=K^{T}>0, \hat{z}$ is the estimate of $z$ and $u_{r}$ will be given.

Then, the estimate of disturbance $\hat{\bar{D}}$ can be written as

$$
\hat{\bar{D}}=\hat{z}+K x_{n}
$$

Based on the output of the designed disturbance observer, $u_{r}$ is designed as

$$
u_{r}=-F(x)-\hat{W}^{T} \varphi(Z)+\tau-\hat{\bar{D}}
$$


Substituting (48) into (45), we obtain

$$
\begin{aligned}
\dot{x}_{n} & =F(x)+u_{r}+W^{* T} \varphi(Z)+\bar{D} \\
& =W^{* T} \varphi(Z)-\hat{W}^{T} \varphi(Z)+\tau+\bar{D}-\hat{\bar{D}} \\
& =-\tilde{W}^{T} \varphi(Z)+\tau+\tilde{\bar{D}}
\end{aligned}
$$

where $\tilde{W}=\hat{W}-W^{*}$.

Considering (28), (29) and (31), (49) can be rewritten as

$$
\dot{e}_{s}=-\gamma e_{s}+\tilde{W}^{T} \Phi(Z)-\tilde{\bar{D}}
$$

Consider the adaptive law for $\hat{W}$ as

$$
\dot{\hat{W}}=-\Lambda\left(\varphi(Z) e_{s}^{T}+\beta \hat{W}\right)
$$

where $\Lambda=\Lambda^{T}>0$ and $\beta>0$.

The above design procedure can be summarized in the following theorem, which contains the adaptive fuzzy control results for the uncertain MIMO system dynamics (3) with system uncertainty, external disturbance, control singularity and unknown input saturation.

Theorem 3 Considering the uncertain MIMO nonlinear system (3) with system uncertainty, external disturbance, control singularity and unknown non-symmetric input saturation, the compound disturbance $\bar{D}$ is approximated using disturbance observer (46) and (47), the updated law of fuzzy system parameter is chosen as (51) and the robust tracking control is designed according to (41) and (48). Then, the tracking error e, the disturbance approximation error $\tilde{D}$ and the parameter $\tilde{W}$ are semi-globally uniformly stable.

The proof of Theorem 2 can be similarly done in accordance with the proof of Theorem 1.

Remark 3 From (44), the effects of control singularity and unknown non-symmetric input saturation are treated as a part of the compound disturbance which is approximated using the nonlinear disturbance observer (46) and (47). Although the uncertain MIMO nonlinear system (3) has the control singularity case and unknown non-symmetric input saturation, lyapunov analysis show that the trajectory of the closed-loop system is asymptotically convergent under the proposed adaptive fuzzy tracking control. To the best of our knowledge, it is the first time in the literature that the control singularity and unknown non-symmetric input saturation are considered in the control design, and as such it is different from the existing results. 


\section{Simulation Results}

Simulation results are given to illustrate the effectiveness of our proposed control techniques in this section. Let us consider the uncertain MIMO nonlinear system in the form of

$$
\begin{aligned}
\dot{x}_{1} & =x_{2} \\
\dot{x}_{2} & =\left[\begin{array}{l}
F_{1}(x) \\
F_{2}(x)
\end{array}\right]+\left[\begin{array}{ll}
g_{11}(x) & g_{12}(x) \\
g_{21}(x) & g_{22}(x)
\end{array}\right] u+\left[\begin{array}{c}
\Delta F_{1}(x) \\
\Delta F_{2}(x)
\end{array}\right]+\left[\begin{array}{l}
d_{1}(x) \\
d_{2}(x)
\end{array}\right] \\
y & =x_{1}
\end{aligned}
$$

where $x=\left[x_{11}, x_{12}, x_{21}, x_{22}\right]^{T}, x_{1}=\left[x_{11}, x_{12}\right]^{T}, x_{2}=\left[x_{21}, x_{22}\right]^{T}, F_{1}(x)=\left(1+x_{11}^{2}\right) x_{21}+x_{12} e^{-0.5 x_{22}}$, $F_{2}(x)=\sin \left(x_{11} x_{22}\right)+x_{21} x_{12}^{2}, g_{11}(x)=-1+\cos \left(x_{12} x_{22}\right)+\sin \left(x_{11} x_{22}\right), g_{12}(x)=1, g_{21}(x)=1-$ $e^{x_{12} x_{22}}, g_{22}(x)=1-x_{12} x_{22} e^{-x_{11} x_{21}}, \Delta F_{1}(x)=0.2 \sin \left(0.1 x_{11} x_{21}\right)+0.3 \sin \left(0.2 x_{12} x_{22}\right), \Delta F_{2}(x)=$ $0.2 \sin \left(0.1 x_{12} x_{21}\right)+0.3 \sin \left(0.2 x_{11} x_{22}\right), d_{1}=0.2 \sin \left(0.5 x_{11} x_{22}\right)+0.3 \sin \left(0.2 \sqrt{t} x_{12} x_{21}\right)$ and $d_{2}=0.3$ $\sin \left(0.25 x_{12} x_{21}\right)+0.2 \sin \left(0.2 \sqrt{t+1} x_{11} x_{22}\right)$.

Obviously, nonlinear system (52) has the same as form of the studied uncertain MIMO nonlinear system (3). Form the expression of $G(x)$, we can see that there exists control singularity case for the nonlinear system (52). Following, the extensive simulation to demonstrate the effectiveness of proposed adaptive fuzzy tracking control. Before the design of nonlinear disturbance observer and adaptive fuzzy tracking control, all design parameters in the simulation study are chosen as $K=200 I_{2 \times 2}, \Lambda=0.01 I_{7 \times 7} \beta=0.002, \gamma=50, k_{1}=200$, and $\delta=0.05$. The initial state conditions are arbitrarily chosen as $x_{11}=0, x_{12}=0.1, x_{21}=-0.1, x_{22}=0.1, \hat{d}_{1}=0, \hat{d}_{2}=0.1$ and $\hat{W}=0.2$. The desired trajectory is taken as $y_{1 d}=0.5(1.6 \sin (t)-\sin (0.5 t))$ and $y_{2 d}=0.5(\sin (t)+\sin (0.5 t))$. For the non-symmetric saturation constraint, the saturation values are given by $u_{1 \max }=1.8, u_{1 \min }=-2.0$, $u_{2 \max }=1.2$ and $u_{2 \min }=-1.5$. In the simulation, fuzzy membership functions are chosen as follows

$$
\begin{array}{rlrl}
\mu_{F_{i}^{1}} & =\exp \left[\frac{-0.5\left(x_{i}+1.5\right)^{2}}{4}\right], & \mu_{F_{i}^{2}}=\exp \left[\frac{-0.5\left(x_{i}+1\right)^{2}}{4}\right], \\
\mu_{F_{i}^{3}}=\exp \left[\frac{-0.5\left(x_{i}+0.5\right)^{2}}{4}\right], & \mu_{F_{i}^{4}}=\exp \left[\frac{-0.5 x_{i}^{2}}{4}\right], \\
\mu_{F_{i}^{5}}=\exp \left[\frac{-0.5\left(x_{i}-0.5\right)^{2}}{4}\right], & \mu_{F_{i}^{6}}=\exp \left[\frac{-0.5\left(x_{i}-1\right)^{2}}{4}\right], \\
\mu_{F_{i}^{7}}=\exp \left[\frac{-0.5\left(x_{i}-1.5\right)^{2}}{4}\right] . & &
\end{array}
$$

The disturbance observer and the adaptive fuzzy tracking control are designed according to (46), (47), (41) and (48). The fuzzy system parameter is updated by (51). The tracking results of the uncertain MIMO nonlinear system (52) with input saturation and control singularity case are shown in Figure 1, 
Figure 2 and Figure 3 under the proposed adaptive fuzzy tracking control. Although, there exist nonsymmetric input saturation, control singularity and time-varying external disturbance, the tracking performance of uncertain MIMO nonlinear system (52) is still satisfactory and tracking errors are asymptotically convergent. The control input signals are shown in Figure 4 and Figure 5. From Figure 4 and Figure 5, the non-symmetric input saturation of control input signals are observed. Norm of the approximation parameter of fuzzy logical system is presented in Figure 6 which shows the convergent ability of approximation parameter.

Based on above simulation results, we obtain that the proposed adaptive fuzzy control technique for uncertain MIMO nonlinear systems using disturbance observer is valid.

\section{Conclusion}

In this paper, adaptive fuzzy tracking control has been proposed for a class of uncertain MIMO nonlinear systems. To improve the ability of disturbance attenuation and closed-loop system performance, the nonlinear disturbance observer has been adopted to estimate the compound disturbance. Using outputs of the disturbance observer and the fuzzy logical system, the disturbance-observerbased adaptive fuzzy tracking control has been proposed for the uncertain MIMO nonlinear system with the non-symmetric input saturation, control singularity and the time-varying external disturbance. The stability of the closed-loop system has been proved using rigorous Lyapunov analysis. Finally, simulation results have been used to illustrate the effectiveness of the proposed adaptive fuzzy tracking control scheme.

\section{References}

[1] M. Makoudi and L. Radouane, "A robust model reference adaptive control for non-minimum phase systems with unknown or time-varying delay," Automatica, vol. 36, no. 7, pp. 1057-1065, 2000.

[2] B. M. Mirkin and P. O. Gutman, "Output feedback model reference adaptive control for MIMO plants with state delay," Systems \& Control Letters, vol. 54, no. 10, pp. 961-972, 2005.

[3] X. D. Tang, G. Tao, and S. M. Joshi, "Adaptive actuator failure compensation for nonlinear MIMO systems with an aircraft control application," Automatica, vol. 43, no. 11, pp. 1869-1883, 2007.

[4] B. Yao and M. Tomizuka, "Adaptive robust control of MIMO nonlinear systems in semi-strict feedback forms," Automatica, vol. 37, no. 9, pp. 1305-1321, 2001. 
[5] S. S. Ge and J. Wang, "Robust adaptive tracking for time varying uncertain nonlinear systems with unknown control coefficients," IEEE Transactions on Automatic Control, vol. 48, no. 8, pp. 1463-1469, 2003.

[6] W. C. Luo, Y. C. Chu, and K. V. Ling, "Inverse optimal adaptive control for attitude tracking of spacecraft," IEEE Transactions on Automatic Control, vol. 50, no. 11, pp. 1639-1654, 2005.

[7] N. V. Q. Hung, H. D. Tuan, T. Narikiyo, and P. Apkarian, "Adaptive control for nonlinearly parameterized uncertainties in robot manipulators," IEEE Transactions on Control System Technology, vol. 16, no. 3, pp. 458-468, 2008.

[8] W. S. Yu and C. J. Sun, "Fuzzy model based adaptive control for a class of nonlinear systems," IEEE Transactions on Fuzzy systems, vol. 9, no. 3, pp. 413-425, 2001.

[9] S. C. Tong, Q. Li, and T. Y. Chai, "Fuzzy adaptive control for a class of nonlinear systems," Fuzzy sets and Systems, vol. 101, no. 1, pp. 31-39, 1999.

[10] S. C. Tong and H. X. Li, "Direct adaptive fuzzy output tracking control of nonlinear systems," Fuzzy sets and systems, vol. 128, no. 1, pp. 107-115, 2002.

[11] W. S. Chen, L. C. Jiao, R. H. Li, and J. Li, "Adaptive backstepping fuzzy control for nonlinearly parameterized systems with periodic disturbances," IEEE Transactions on Fuzzy Systems, vol. 18, no. 4, pp. 674-685, 2010 .

[12] W. S. Chen and Z. Q. Zhang, "Globally stable adaptive backstepping fuzzy control for output-feedback systems with unknown high-frequency gain sign," Fuzzy Sets and Systems, vol. 161, no. 6, pp. 821-836, 2010.

[13] C. L. Hwang and C. Y. Kuo, "A Stable adaptive fuzzy sliding-mode control for affine nonlinear systems with application to four-bar linkage systems," IEEE Transactions on Fuzzy Systems, vol. 9, no. 2, pp. 238$252,2001$.

[14] G. Rigatos and S. Tzafestas, "Adaptive fuzzy control for the ship steering problem," Mechatronics, vol. 16, no. 3, pp. 479-489, 2006.

[15] S. C. Tong and H. X. Li, "Fuzzy adaptive sliding-mode control for MIMO nonlinear Systems," IEEE Transactions on Fuzzy Systems, vol. 11, no. 3, pp. 354-360, 2003.

[16] S. C. Zhou, G. Feng, and C. B. Feng, "Robust control for a class of uncertain nonlinear systems: adaptive fuzzy approach based on backstepping," Fuzzy Sets and Systems, vol. 151, no. 1, pp. 1-20, 2005.

[17] Y. S. Yang and J. S. Ren, "Adaptive fuzzy robust tracking controller design via small gain approach and its application," IEEE Transactions on Fuzzy Systems, vol. 11, no. 6, pp. 783-795, 2003.

[18] J. H. Park and S. H. Kim, "Direct adaptive output-feedback fuzzy controller for a nonaffine nonlinear system," IEE Proceeding of Control Theory 83 Application, vol. 151, no. 1, pp. 65-72, 2004. 
[19] W. J. Wang and H. R. Lin, "Fuzzy control design for the trajectory tracking on uncertain nonlinear systems," IEEE Transactions on Fuzzy Systems, vol. 7, no. 1, pp. 53-62, 1999.

[20] Y. C. Chang, "Adaptive fuzzy-based tracking control for nonlinear SISO systems via VSS and $H_{\infty}$ approaches," IEEE Transactions on Fuzzy Systems, vol. 9, no. 2, pp. 278-292, 2001.

[21] Y. J. Liu, W. Wang, S. C. Tong, and Y. S. Liu, "Robust adaptive tracking control for nonlinear systems based on bounds of fuzzy approximation parameters," IEEE Transactions on System, Man, And Cybernetics - Part A: System and Humans, vol. 40, no. 1, pp. 170-184, 2010.

[22] Y. J. Liu, W. Wang, and S. C. Tong, "Adaptive fuzzy output tracking control for a class of uncertain nonlinear systems," Fuzzy Sets and Systems, vol. 160, no. 19, pp. 2727-2754, 2009.

[23] Z. G. Sun, N. C. Cheung, S. W. Zhao, and W. C. Gan, "The application of disturbance observerbased sliding mode control for magnetic levitation systems," Proceedings of the Institution of Mechanical Engineers, Part C: Journal of Mechanical Engineering Science, vol. 224, no. 8, pp. 1635-1644, 2010.

[24] E. Kim, "A fuzzy disturbance observer and its application to control," IEEE Transaction on Fuzzy Systems, vol. 10, no. 1, pp. 77-84, 2002.

[25] E. Kim, "A discrete-time fuzzy disturbance observer and its application to control," IEEE Transaction on Fuzzy Systems, vol. 11, no. 3, pp. 399-410, 2003.

[26] W. H. Chen, "Disturbance observer based control for nonlinear systems," IEEE Transaction on Mechatronics, vol. 9, no. 4, pp. 706-710, 2004.

[27] W. H. Chen, D. J. Ballance, P. J. Gawthrop, and J. O'Reilly, "Nonlinear pid predictive controller," IEE Proc. Control Theory Appl., vol. 146, no. 6, pp. 603-611, 1999.

[28] X. K. Chen, C. Y. Su, and T. Fukuda, "A nonlinear disturbance observer for multivariable systems and its application to magnetic bearing systems," IEEE Transaction on Control Systems Technology, vol. 12, no. 4, pp. 569-577, 2004.

[29] W. H. Chen, D. J. Ballance, P. J. Gawthrop, and J. O'Reilly, "A nonlinear disturbance observer for robotic manipulators," IEEE Transaction on Industrial Electronics, vol. 47, no. 4, pp. 932-938, 2000.

[30] X. J. Wei, H. F. Zhang, and L. Guo, "Composite disturbance-observer-based control and terminal sliding mode control for uncertain structural systems," International Journal of Systems Science, vol. 40, no. 10, pp. 1009-1017, 2009.

[31] W. H. Chen, "Nonlinear disturbance observer-enhanced dynamical inversion control of missiles," Journal of Guidance, Control, and Dynamics, vol. 26, no. 1, pp. 161-166, 2003.

[32] L. Guo and W. H. Chen, "Disturbance attenuation and rejection for systems with nonlinearity via DOBC approach," International Journal of Robust and Nonlinear Control, vol. 15, no. 3, pp. 109-125, 2005. 
[33] X. J. Wei and L. Guo, "Composite disturbance-observer-based control and $H_{\infty}$ control for complex continuous models," International Journal of Robust and Nonlinear Control, vol. 20, no. 1, pp. 106-118, 2009.

[34] X. J. Wei and L. Guo, "Composite disturbance-observer-based control and terminal sliding mode control for non-linear systems with disturbances ," International Journal of Control, vol. 82, no. 6, pp. 1082-1098, 2009.

[35] M. Chen, C. S. Jiang, B. Jiang, and Q. X. Wu, "Sliding mode synchronization controller design with neural network for uncertain chaotic systems," Chaos, Solitons E Fractals, vol. 39, no. 4, pp. 1856-1863, 2009.

[36] M. Chen and W. H. Chen, "Sliding mode controller design for a class of uncertain nonlinear system based disturbance observer," Internation al Journal of Adaptive Control and Signal Processing, vol. 24, no. 1, pp. $51-64,2010$

[37] M. Chen and W. H. Chen, "Disturbance observer based robust control for time delay uncertain systems," International Journal of Control, Automation and Systems, vol. 8, no. 2, pp. 445-453, 2010.

[38] M. Chen, S. S. Ge, and B. How, "Robust adaptive neural network control for a class of uncertain MIMO nonlinear systems with input nonlinearities," IEEE Transactions on Neural Networks, vol. 21, no. 5, pp. $796-812,2010$

[39] M. Chen, S. S. Ge, and Y. S. Choo, "Neural network tracking control of ocean surface vessels with input saturation," Proceedings of the IEEE International Conference on Automation and Logistics, pp. 85-89, June, 2009.

[40] J. R. Azinheira and A. Moutinho, "Hover control of an UAV with backstepping design including input saturations," IEEE Transactions on Control Systems Technology, vol. 16, no. 3, pp. 517-526, 2008.

[41] Y. Y. Cao and Z. L. Lin, "Robust stability analysis and fuzzy-scheduling control for nonlinear systems subject to actuator saturation," IEEE Transactions on Fuzzy Systems, vol. 11, no. 1, pp. 57-67, 2003.

[42] Y. S. Zhong, "Globally stable adaptive system design for minimum phase SISO plants with input saturation," Automatica, vol. 41, no. 11, pp. 1539-1547, 2005.

[43] Q. L. Hu, G. F. Ma, and L. H. Xie, "Robust and adaptive variable structure output feedback control of uncertain systems with input nonlinearity," Automatica, vol. 44, no. 4, pp. 552-559, 2008.

[44] S. S. Ge and C. Wang, "Adaptive neural control of uncertain MIMO nonlinear systems," IEEE Transactions on Neural Networks, vol. 15, no. 3, pp. 674-692, 2004.

[45] C. Kwan and F. L. Lewis, "Robust backstepping control of nonlinear systems using neural networks," IEEE Transactions on System, Man, And Cybernetics - Part A: System and Humans, vol. 30, no. 6, pp. 753-766, 2000. 


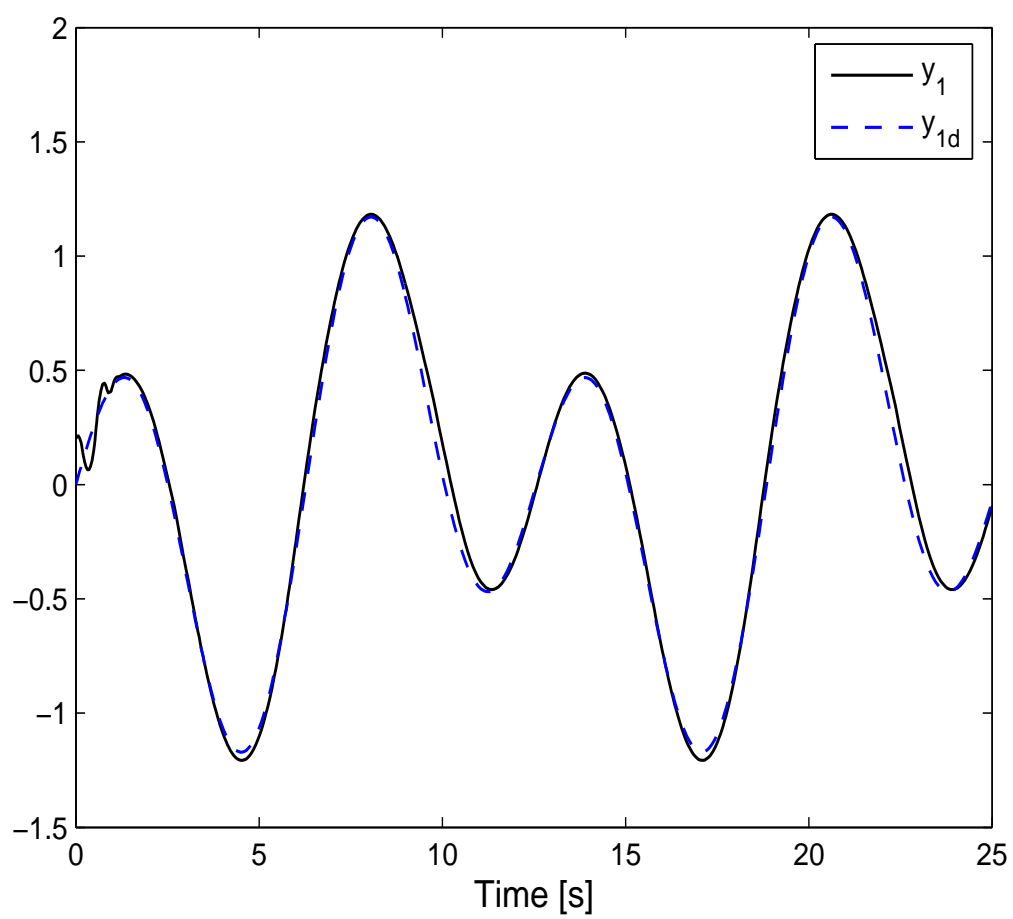

Figure 1: Tracking result of $y_{1}$ and $y_{1 d}$



Figure 2: Tracking result of $y_{2}$ and $y_{2 d}$ 


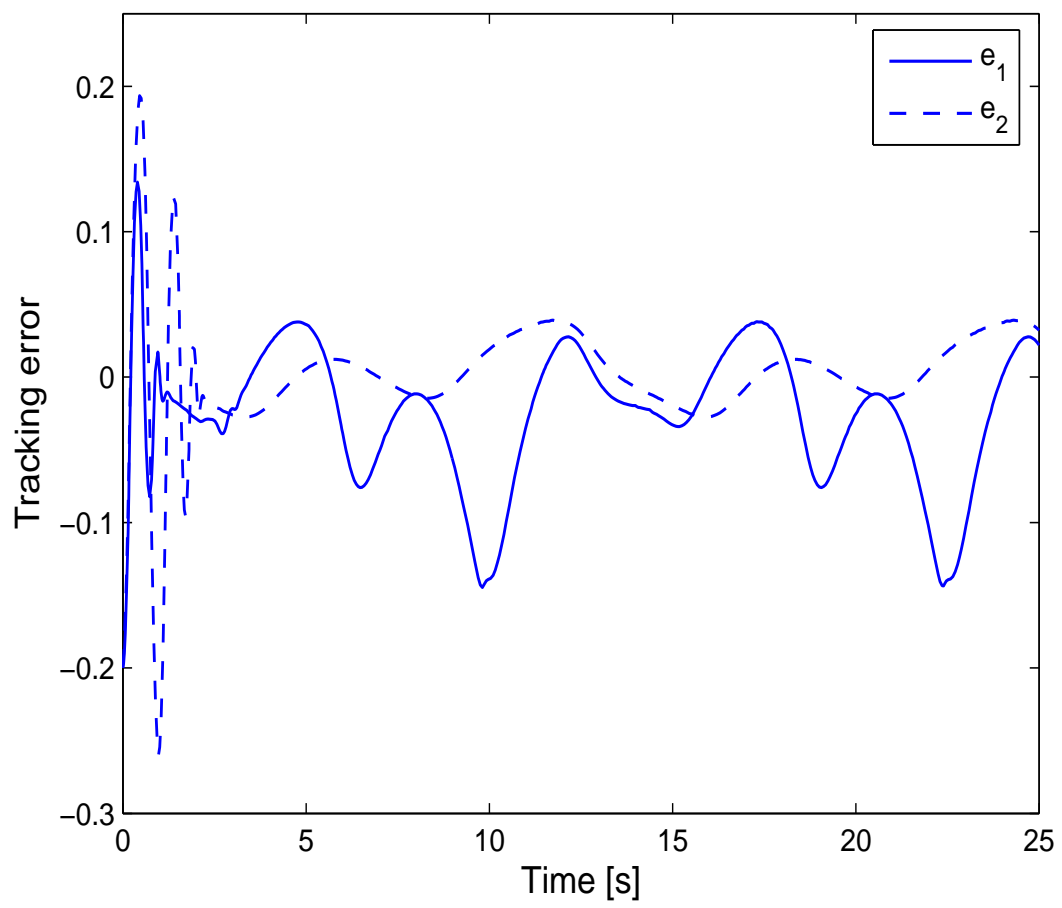

Figure 3: Tracking errors

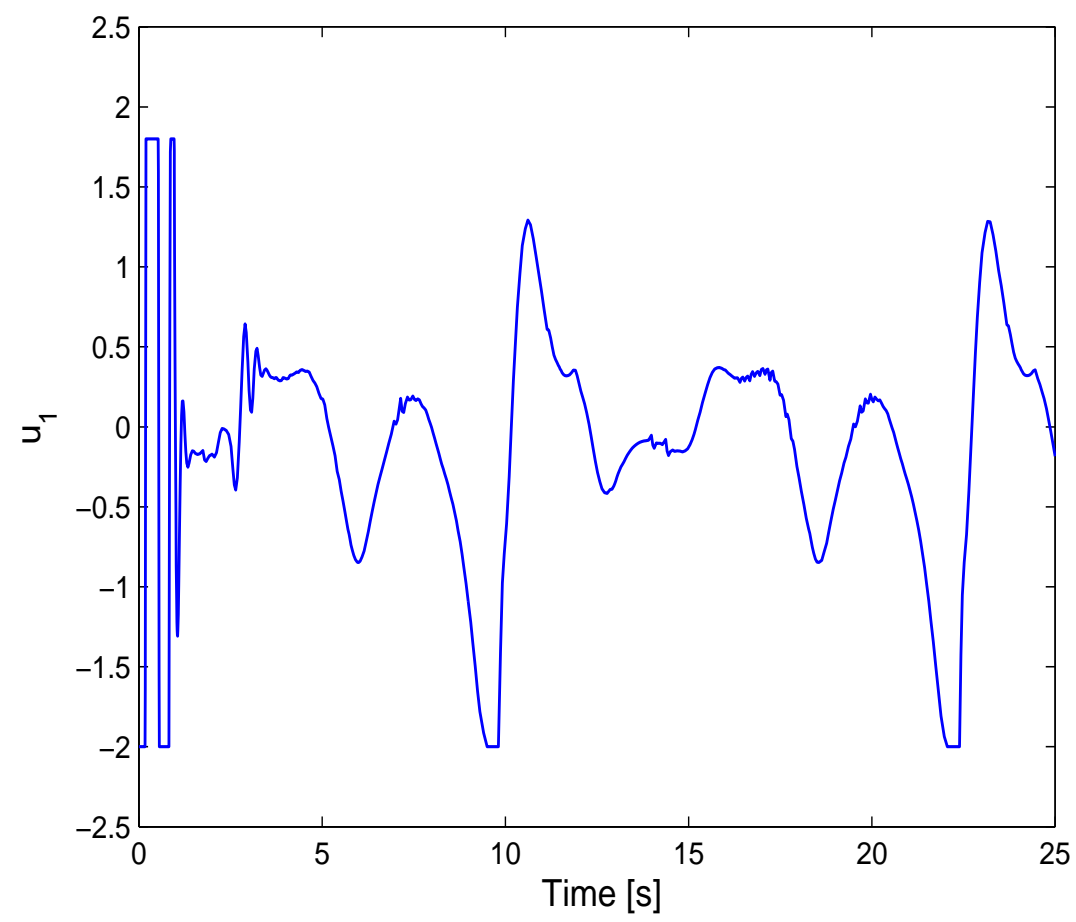

Figure 4: Control input $u_{1}$ 


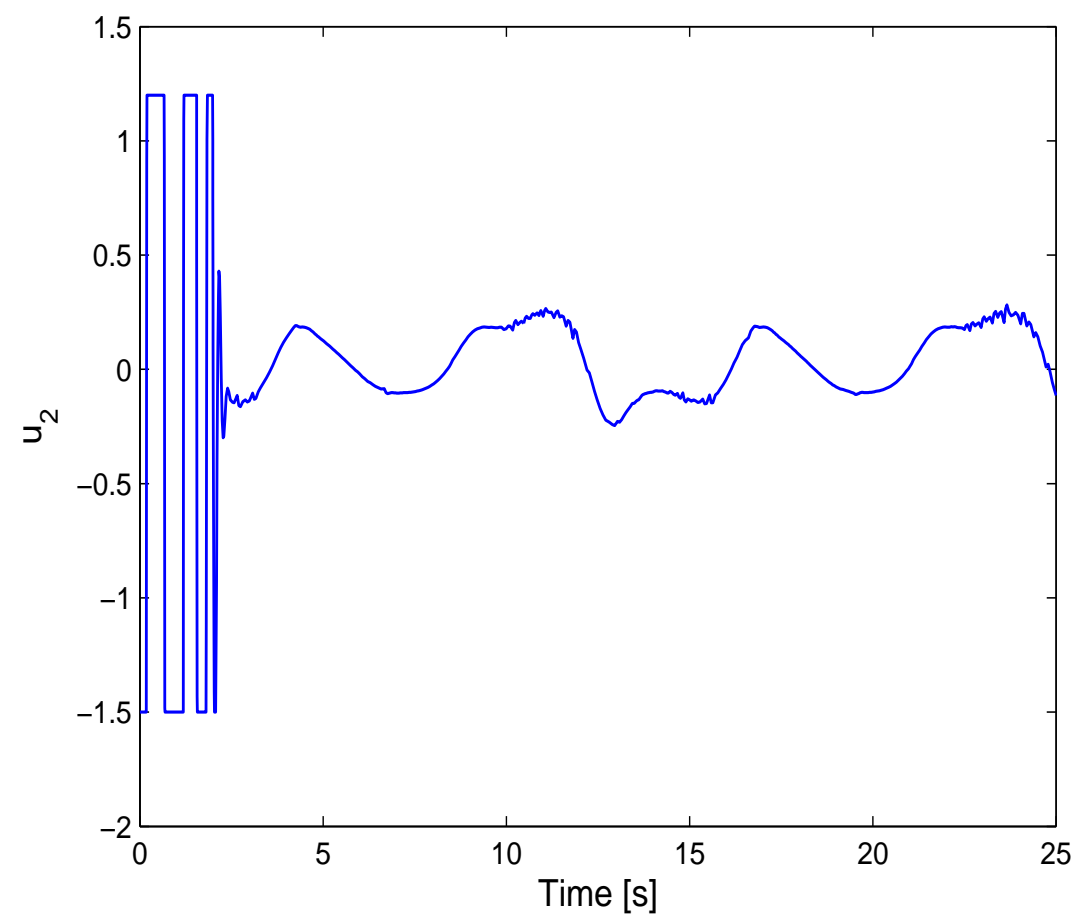

Figure 5: Control input $u_{2}$



Figure 6: Norm of the approximation parameter of fuzzy logical system 